\title{
Beamforming and Scheduling Strategies for Time Slotted Multiuser MIMO Systems
}

\author{
Semih Serbetli and Aylin Yener \\ Electrical Engineering Department \\ The Pennsylvania State University \\ University Park, PA 16802 \\ serbetli@psu.edu \\ yener@ee.psu.edu
}

\begin{abstract}
We investigate the problem of scheduling for the uplink of a time slotted multiuser MIMO system with sum capacity as the performance metric. We first consider scheduling users' transmissions for fixed transmit beamformers and construct a low complexity scheduling algorithm. Since the performance of the system depends on the transmit beamformers, we consider transmit beamformer selection next, and combine the proposed scheduling algorithm with perfectly controlled transmit beamforming, eigen transmit beamforming, and antenna selection. We observe that as the feedback level is increased, the performance of the scheduling algorithm is improved. In particular, a substantial gain is attained when the individual channel state information (CSI) is available at the transmitter side.
\end{abstract}

\section{INTRODUCTION}

Considering the rapidly increasing demand for high data rate and reliable wireless communications, spectrally efficient multiuser transmission schemes are of great importance for next generation wireless multiaccess systems. Recently, the use of multiple antennas at both the transmitter and the receiver side has attracted attention due to their potential to increase the spectral efficiency significantly [1]. There has been considerable research in exploiting the space dimension through transmit diversity, space-time coding and spatial multiplexing for MIMO systems that employ multiple transmit and/or receive antennas [2], [3].

The substantial capacity offered by the single user multiple antenna (MIMO) systems [1] motivates the use of multiple antennas as a means to provide multiple access capabilities [4], [5] or improve the multiple access capabilities of existing multiaccess schemes. In this paper, we consider the latter and assume a time slotted multiuser system where each user as well as the common receiver is equipped with multiple antennas. Employing multiple antennas provides the opportunity to allocate the same time slot to different users, and hence, improves the system performance as compared to TDMA. It is meaningful therefore, to investigate the extent of the potential system performance improvement to be obtained via joint optimization of the users' temporal and spatial transmit strategies.

Our aim in this work is to design scheduling algorithms (temporal transmit strategies) and transmit beamformers (spatial transmit strategies) for time slotted multiuser MIMO systems. The performance metric we consider is the information theoretic sum capacity of the system. Reference [6] studies the sum capacity optimization of SDMA/TDMA, and points out that the slot allocation problem is NP complete. Since exponential complexity is unacceptable for practical systems, scheduling algorithms that require less complexity but achieving near maximum sum capacity are needed.

Our approach relies on viewing the time slotted multiuser MIMO system as a special case of a CDMA system with constrained signatures. Using this observation, we can develop a simple upper bound on the sum capacity of the system under any given scheduling algorithm, including the optimum scheduler. We can then devise scheduling algorithms that aim to approach the upper bound on the capacity. The motivation behind this is that, we would attain near-optimum scheduler capacity, if the scheduler we design results in a sum capacity near the upper bound of the optimum scheduler sum capacity.

First, we consider the case of a multiuser MIMO system with fixed transmit beamforming vectors, and devise a scheduling algorithm. Since the performance of the scheduler is a function of the spatial transmitters of the users, we next tackle the problem of designing the transmit beamformers for different levels of feedback available at the transmit side of each user. We propose several methods for selecting the spatial transmit beamformers in accordance with different levels of feedback available at the transmit side. Our results suggest that joint design of transmit beamformers and the scheduler improves the performance and better sum capacity is attained with increased level of feedback at the transmit side.

\section{System Model}

We consider the uplink of a single cell synchronous multiuser MIMO system with $K$ users and $N$ time slots. The common receiver is equipped with $N_{R}$ receive antennas and user $j$ has $N_{T_{j}}$ transmit antennas. We assume that the $j$ th user transmits its symbol by precoding it with an $N_{T_{j}} \times 1$ unit norm spatial transmit beamforming vector, $\mathbf{f}_{j}$ in the time slot allocated. We assume that the spatial transmit beamforming vector for each user is given and fixed first. We relax this assumption later and investigate the impact of spatial transmit beamforming. Similar to the notation in [3], [6], the received vector in each time slot $i$ is

$$
\mathbf{r}_{i}=\sum_{j \in \mathcal{K}_{i}} \sqrt{P_{j}} \mathbf{H}_{j} \mathbf{f}_{j} s_{j}+\mathbf{n}_{i}, \quad i=1, \ldots, N
$$


where $P_{j}, s_{j}$ and $\mathbf{H}_{j}$ are the transmit power, symbol, and the $N_{R} \times N_{T_{j}}$ complex MIMO channel matrix of user $j$, respectively. $\mathbf{n}_{i}$ is the zero mean complex Gaussian noise vector in the $i$ th time slot with $E\left[\mathbf{n}_{i} \mathbf{n}_{i}^{\dagger}\right]=\sigma^{2} \mathbf{I}$ where $(\cdot)^{\dagger}$ denotes the Hermitian of a vector or matrix. $\mathcal{K}_{i}$ denotes the set of users that are assigned to the $i$ th time slot with each set satisfying $\mathcal{K}_{i} \bigcap \mathcal{K}_{l}=\emptyset, \forall i \neq l$ and $\bigcup_{i=1}^{N} \mathcal{K}_{i}=\{1,2, \ldots, K\}$. We assume that the channels are flat fading and the channel realizations are constant over a frame of coded symbols, and perfectly known at the receiver side. For clarity of exposition, we denote the joint effect of the transmit power, channel matrix and spatial transmit beamforming vector of user $j$ as $\mathbf{a}_{j}=\sqrt{P_{j}} \mathbf{H}_{j} \mathbf{f}_{j}$ which forms a spatial signature for user $j$. The received vector in each time slot can be represented as:

$$
\mathbf{r}_{i}=\sum_{j \in \mathcal{K}_{i}} \mathbf{a}_{j} s_{j}+\mathbf{n}_{i}, \quad i=1, \ldots, N
$$

Defining $\left(N N_{R}\right) \times N$ block diagonal matrices $\left\{\mathbf{A}_{j}\right\}$ with $\mathbf{a}_{j}$ 's as the block diagonal entries, and stacking all the received signals at each time slot, the received signal can be represented as

$$
\mathbf{r}=\sum_{j=1}^{K} \mathbf{A}_{j} \mathbf{t}_{j} s_{j}+\mathbf{n} \quad \text { with } \mathbf{t}_{j}=\mathbf{e}_{i} \text { if } j \epsilon \mathcal{K}_{i}
$$

Here $\mathbf{e}_{i}$ is an $N \times 1$ vector with $N-1$ zeros and 1 in the $i$ th entry. Throughout the paper, we consider multiuser MIMO systems with $K \leq N N_{R}$ users and develop scheduling algorithms for such systems.

\section{Sum CAPACITY AND ITS UPPER BOUND}

Our aim in this section is to investigate the effect of scheduling on the sum capacity of multiuser MIMO systems and to describe our approach for developing near optimum scheduling algorithms.

Previous work showed that the information theoretic sum capacity of a multiuser MIMO scheduler in the time slot $i$ with effective signatures $\left\{\mathbf{a}_{j}=\sqrt{P_{j}} \mathbf{H}_{j} \mathbf{f}_{j}\right\}$ is given by [7]

$$
C_{\mathcal{K}_{i}}=\frac{1}{2 N} \log \left[\operatorname{det}\left(\mathbf{I}_{N_{R}}+\sigma^{-2} \sum_{j \in \mathcal{K}_{i}} \mathbf{a}_{j} \mathbf{a}_{j}^{\dagger}\right)\right]
$$

Formally, the sum capacity optimization problem for multiuser MIMO scheduling is given by

$$
\begin{gathered}
\max _{\mathcal{K}_{i}} C_{\text {sum }}=\sum_{i=1}^{N} C_{\mathcal{K}_{i}} \\
\text { s.t. } \bigcup_{i=1}^{N} \mathcal{K}_{i}=\{1,2, \ldots, K\}, \quad \mathcal{K}_{i} \bigcap \mathcal{K}_{l}=\emptyset, \quad \forall i \neq l
\end{gathered}
$$

The sum capacity maximization for multiuser MIMO scheduling is a combinatorial optimization problem and has been recently studied in the context of SDMA/TDMA systems where it has been pointed out to be NP-complete [6]. As expected, the globally optimum schedule improves the sum capacity of the SDMA/TDMA systems significantly [6]. However, obviously, the associated complexity would render the optimum scheduler impractical even for a moderate number of users: simpler scheduling algorithms that achieve near maximum sum capacity are needed.

From a system point of view, the time slotted multiuser MIMO system can be viewed as a CDMA system with a processing gain $N N_{R}$ with structurally constrained signatures $\left\{\mathbf{b}_{j}=\mathbf{A}_{j} \mathbf{t}_{j}\right\}$. The received signal power of user $j$ is simply $\left\|\mathbf{a}_{j}\right\|^{2}$ for the multiuser MIMO scheduler. The sum capacity of CDMA systems with users having arbitrary received powers is studied and the optimum signature sequences are identified [8]. Since this case corresponds to relaxation of signature constraints, it constitutes an upper bound on the sum capacity of the optimum multiuser MIMO scheduler. For a multiuser MIMO scheduler with $K \leq N N_{R}$, the upper bound is the sum capacity of an underloaded CDMA system which employs orthogonal effective signature sequences. In particular, the sum capacity upper bound $C_{\text {upper }}$ is

$$
C_{\text {sum }} \leq C_{\text {upper }}=\frac{1}{2 N} \sum_{j=1}^{K} \log \left[1+\sigma^{-2}\left\|\mathbf{a}_{j}\right\|^{2}\right]
$$

Given this observation, and the fact that the sum capacity of the optimum scheduler does not have a closed form, we try to design scheduling strategies to approach the bound instead. The rationale is that if the schedulers we design yield performance near the upper bound, their performance must be closer to that of the optimum scheduler. Hence, without computing the exact maximum sum capacity of multiuser MIMO scheduler, one can investigate the performance of a given scheduling algorithm by comparing with the upper bound [9].

\section{Multiuser MiMO SCHEduler}

Our aim in this section is to design an algorithm that schedules each user to a time slot for fixed beamformers and achieves sum capacity near the upper bound developed in the previous section.

As pointed out in the previous section, the optimum unconstrained effective signatures for $N N_{R} \geq K$, are the orthogonal signature sequences. Thus, the near-optimum scheduling strategy should try to assign the slots to users such that the effective signatures, $\left\{\mathbf{A}_{j} \mathbf{t}_{j}\right\}$ are as close to being orthogonal as they can. Observe that assigning more than $N_{R}$ users to the same time slot is likely to cause high correlation among the users. In addition to the fact that each time slot should not be assigned more than $N_{R}$ users, intuitively, it is logical to assign no more than $\left\lceil\frac{\text { Number of users }}{\text { Number of time slots }}\right\rceil \leq N_{R}$ users to each time slot for the sake of fairness.

The above observations suggest that an $N$ step sequential user assignment algorithm that tries to select the spatially less correlated users for each time slot is a good candidate for near optimum performance. Specifically, at each step, the number of users that will be assigned for time slot is $\left\lceil\frac{\text { Number of available users }}{\text { Number of available time slots }}\right\rceil$ which is guaranteed not to exceed $N_{R}$ users for $N N_{R} \geq K$.

Assume that user $k$ is transmitting in the $i$ th time slot. Let us define the set of users in slot $i$ excluding user $k$ as $\mathcal{K}_{i}-\{k\}=$ 
TABLE I

Sum Capacity Based Sequential Scheduling Algorithm

\begin{tabular}{|ll|}
\hline System & Parameters \\
$\mathcal{K}_{a}$ & : Available users that are not assigned to a time slot \\
$\mathcal{K}_{i}$ & : The users assigned to the time slot $i=1, \ldots N$ \\
$\left\{\mathbf{a}_{j}\right\}$ & : Effective spatial signatures of users \\
Avuser & : No. of users that will be assigned to the time slot \\
Scheduling & Algorithm \\
$\mathcal{K}_{a}$ & $=\{1,2, \ldots, K\}$ \\
For & $i=1: N$ \\
& User Selection for time slot i \\
& Avuser $=\left\lceil\frac{n\left(\mathcal{K}_{a}\right)}{N-i+1}\right\rceil$ \\
& For $j=1:$ Avuser \\
& $k^{\star}=\underset{k \in \mathcal{K}_{a}}{\arg z_{i k}}$ \\
& $\mathcal{K}_{i}=\mathcal{K}_{i} \bigcup\left\{k^{\star}\right\}$ \\
& $\mathcal{K}_{a}=\mathcal{K}_{a} \backslash\left\{k^{\star}\right\}$ \\
& End \\
&
\end{tabular}

$\overline{\mathcal{K}}_{i}^{(k)}$. The contribution of user $k$ on the sum capacity is simply

$$
\begin{aligned}
\Delta_{i k} & =C_{\mathcal{K}_{i}}-C_{\overline{\mathcal{K}}_{i}^{(k)}} \\
& =\frac{1}{2 N} \log \left[1+\mathbf{a}_{k}^{\dagger}\left(\sigma^{2} \mathbf{I}_{N_{R}}+\sum_{j \in \overline{\mathcal{K}}_{i}^{(k)}} \mathbf{a}_{j} \mathbf{a}_{j}^{\dagger}\right)^{-1} \mathbf{a}_{k}\right]
\end{aligned}
$$

Also, the capacity of user $k$ with its unconstrained effective signature orthogonal to the other users is

$$
C_{k}=\frac{1}{2 N} \log \left(1+\sigma^{-2}\left\|\mathbf{a}_{k}\right\|^{2}\right)
$$

Observe that the assignment of user $k$ to time slot $i$ will result in a difference of $C_{k}-\Delta_{i k}$ between the sum capacity upper bound, and the achieved sum capacity of the multiuser MIMO scheduler from user $k$ 's perspective. This difference can be expressed as

$$
C_{k}-\Delta_{i k}=\frac{1}{2 N} \log \frac{1+\sigma^{-2}\left\|\mathbf{a}_{k}\right\|^{2}}{1+\mathbf{a}_{k}^{\dagger}\left(\sigma^{2} \mathbf{I}_{N_{R}}+\sum_{j \in \overline{\mathcal{K}}_{i}^{(k)}} \mathbf{a}_{j} \mathbf{a}_{j}^{\dagger}\right)^{-1} \mathbf{a}_{k}}
$$

Thus, the user with the highest

$$
z_{i k}=\frac{1+\mathbf{a}_{k}^{\dagger}\left(\sigma^{2} \mathbf{I}_{N_{R}}+\sum_{j \in \overline{\mathcal{K}}_{i}^{(k)}} \mathbf{a}_{j} \mathbf{a}_{j}^{\dagger}\right)^{-1} \mathbf{a}_{k}}{1+\sigma^{-2}\left\|\mathbf{a}_{k}\right\|^{2}}
$$

will result in the smallest difference from the upper bound from a single user's perspective. Approaching the sum capacity maximization problem for the multiuser MIMO scheduler as a sequential user/slot assignment problem, we choose to minimize the difference between the sum capacity of the unconstrained effective signature upper bound and the achieved sum capacity of the multiuser MIMO scheduler from a single user's perspective. That is, at each user/slot assignment step, we choose the user that has the highest $z_{i k}$ to assign to time slot $i$. Observe that the algorithm is greedy in nature and completes the assignment slot by slot. Observe also that although later slots have a smaller pool of users to choose from, the earlier slots may need to carry one more user depending on the total number of users in the system. Notice that the algorithm has no preference for the first user to be assigned to a time slot. Thus, an arbitrary user can be chosen from the available users. The algorithm proposed is summarized in Table I.

\section{Transmit Beamformer Design with Different LEVELS OF FEEDBACK}

The previous section considered the case where the spatial transmit beamforming vectors are fixed inputs for the multiuser MIMO scheduler. It is clear that the scheduling algorithm is driven by the effective spatial transmit strategies, $\left\{\mathbf{a}_{j}=\right.$ $\left.\sqrt{P_{j}} \mathbf{H}_{j} \mathbf{f}_{j}\right\}$ of the users. That is, the performance of the multiuser MIMO system with the near-optimum scheduler, is a function of the choice of the transmit beamformers $\left\{\mathbf{f}_{j}\right\}$. In turn, the choice of the transmit beamformers is highly dependent on the feedback level at the transmitter side. We consider next different levels of feedback at the transmitter side, and derive the best corresponding transmit beamformers to be employed jointly with the multiuser MIMO scheduler. Specifically, perfect transmit beamforming feedback, individual CSI feedback, and antenna selection feedback cases are investigated next.

\section{A. Perfect Transmit Beamforming Feedback}

In this section, we assume that we have a reliable and an error free feedback channel to the transmitter side and that we will design beamformers for this system. The motivation for this design is to obtain a performance upper bound for that of any beamformer design with limited feedback.

In the development of the scheduling algorithm, the time slot allocation is done by considering the performance in terms of minimizing the gap between the sum capacity of the multiuser MIMO scheduler and the upper bound from a single user's perspective. Using (9) and $\left\{\mathbf{a}_{j}=\sqrt{P_{j}} \mathbf{H}_{j} \mathbf{f}_{j}\right\}_{j=1}^{K}$, the effect of the spatial transmit beamforming vectors on the sum capacity of a time slot can be expressed as

$$
\Delta_{i k}=\frac{1}{2 N} \log \left[1+P_{k} \mathbf{f}_{k}^{\dagger} \mathbf{H}_{k}^{\dagger}\left(\sigma^{2} \mathbf{I}_{N_{R}}+\sum_{j \in \overline{\mathcal{K}}_{i}^{(k)}} \mathbf{a}_{j} \mathbf{a}_{j}^{\dagger}\right)^{-1} \mathbf{H}_{k} \mathbf{f}_{k}\right]
$$

Recall that the sum capacity upper bound in (7) is dependent on the received powers of users, i.e., $\left\{\left\|\mathbf{a}_{k}\right\|^{2}=\right.$ $\left.P_{k} \mathbf{f}_{k}^{\dagger} \mathbf{H}_{k}^{\dagger} \mathbf{H}_{k} \mathbf{f}_{k}\right\}$. Notice that the received power of user $k$ is maximized when the maximum eigenvalued eigenvector of $\mathbf{H}_{k}^{\dagger} \mathbf{H}_{k}$ is used as a transmit beamforming vector, and the upper bounds for the sum capacity can be reformulated as

$$
C_{k}=\frac{1}{2} \log \left(1+\sigma^{-2} P_{k} \max _{\left\{\mathbf{f} \mid \mathbf{f}^{\dagger} \mathbf{f}=1\right\}} \mathbf{f}^{\dagger} \mathbf{H}_{k}^{\dagger} \mathbf{H}_{k} \mathbf{f}\right)
$$

resulting in the metric used in the user/slot assignment, $z_{i k}$

$$
z_{i k}\left(\mathbf{f}_{k}\right)=\frac{1+P_{k} \mathbf{f}_{k}^{\dagger} \mathbf{H}_{k}^{\dagger}\left(\mathbf{I}_{N_{R}}+\sigma^{-2} \sum_{j \epsilon \overline{\mathcal{K}}_{i}^{(k)}} \mathbf{a}_{j} \mathbf{a}_{j}^{\dagger}\right)^{-1} \mathbf{H}_{k} \mathbf{f}_{k}}{1+\sigma^{-2} P_{k} \max _{\left\{\mathbf{f} \mid \mathbf{f}^{\dagger} \mathbf{f}=1\right\}} \mathbf{f}^{\dagger} \mathbf{H}_{k}^{\dagger} \mathbf{H}_{k} \mathbf{f}}
$$

We observe that the transmit beamforming vector that maximizes $z_{i k}$ is the maximum eigenvalued eigenvector of $\mathbf{H}_{k}^{\dagger}\left(\sigma^{2} \mathbf{I}_{N N_{R}}+\sum_{j \in \overline{\mathcal{K}}_{i}^{(k)}} \mathbf{a}_{j} \mathbf{a}_{j}^{\dagger}\right)^{-1} \mathbf{H}_{k}$. Thus, the scheduling algorithm with perfect transmit beamforming feedback (PTBF) should compare the performance of the users with their best 
transmit beamforming vectors at each step. The resulting scheduling and beamformer design algorithm is a two-step algorithm. The best transmit beamformers are found at the first step, and the user with the highest $z_{i k}$ is assigned a time slot next. After the assignment of the time slot, the users may choose to update their transmit beamformers improving the performance at each step. In the numerical results, we have chosen to perform a single iteration in order to provide a fair comparison with the limited feedback designs given in the next two sections, i.e., eigen mode and antenna selection.

\section{B. Eigen Mode Selection}

In the case of limited channel state feedback, a feasible scenario is that each user knows its own channel. This is a reasonable assumption when the system is operated in time division duplex. In this approach, the transmit beamformer vector of each user can be chosen among the set of eigenvectors of the channel matrices, $\left\{\mathbf{H}_{k}^{\dagger} \mathbf{H}_{k}\right\}$, and the only required feedback between the user and the receiver is which eigen modes should be used. Consider the case where one eigen mode is selected. When user $k$ selects eigen mode $m, \mathbf{a}_{k}=\sqrt{P_{k}} \mathbf{H}_{k} \mathbf{u}_{k m}$ where $\mathbf{u}_{k m}$ is the $m$ th eigen vector of $\mathbf{H}_{k}^{\dagger} \mathbf{H}_{k}$. It is clear that the received power of the user $k,\left\|\mathbf{a}_{k}\right\|^{2}=P_{k} \mathbf{u}_{k m}^{\dagger} \mathbf{H}_{k}^{\dagger} \mathbf{H}_{k} \mathbf{u}_{k m}$, is maximized when the maximum eigenvalued eigen mode is used. In this case, the single user performance bound, $C_{k}$ for the eigen mode selection remains the same as in perfect transmit beamforming case (14).

Observe from (15) that, the metric is both dependent on the received power and the effective spatial signatures of the users that are already assigned. Assuming that the scheduler will eventually allocate the users with spatially low correlations to the same time slot, intuitively, an effective eigen mode selection method is to choose the eigen mode that will maximize the received power of the user, i.e., the maximum eigenvalued eigen mode. In this case, the transmit beamformer and the effective spatial signature of user $k$ become

$\mathbf{f}_{k}=\underset{\mathbf{u}_{k m} \mid m \epsilon\left\{1,2, \ldots, N_{T_{k}}\right\}}{\arg \max } \mathbf{u}_{k m}^{\dagger} \mathbf{H}_{k}^{\dagger} \mathbf{H}_{k} \mathbf{u}_{k m} ; \mathbf{a}_{k}=\sqrt{P_{k}} \mathbf{H}_{k} \mathbf{f}_{k}$

The scheduling algorithm with maximum received power eigenmode selection (MAXES) is the algorithm presented in Table I with the spatial signatures defined as the spatial signatures with the maximum eigenvalued eigenmodes.

MAXES is expected to perform well especially if one eigenvalue is significantly larger than the others, and the MIMO channels of the users are independent of each other. On the other hand, if the channels of the users are highly correlated, choosing the eigenmode that maximizes the received power may not significantly outperform the remaining eigenmodes. In that case, we may choose to employ a scheduling algorithm that considers each eigenmode, and chooses the eigenmode of the user that has the best performance in the sense of minimizing the performance difference from a single user's perspective. We term this algorithm the scheduling algorithm with generalized eigenmode selection (GENES). The algorithm with generalized eigenmode selection is a two-step algorithm where the best eigenmodes are found at the first step, and the user with the highest $z_{i k}$ is assigned a time slot next.

\section{Antenna Selection}

An alternative approach for limited feedback is antenna selection, where the only required feedback is which antenna(s) should be used [10], [11]. Consider the case where one transmitter antenna is selected. In this case, the spatial signature of the user is the spatial signature of the transmitter antenna to be used, i.e., when user $k$ selects transmitter antenna $m, \mathbf{a}_{k}=\sqrt{P_{k}} \mathbf{h}_{k m}$ where $\mathbf{h}_{k m}$ is the $m$ th column vector of user $k$ 's channel matrix. It is clear that the received power of the user $k$ is $\left\|\mathbf{a}_{k}\right\|^{2}=P_{k}\left\|\mathbf{h}_{k m}\right\|^{2}$. For antenna selection, the single user performance upper bound is defined by the maximum received power of the transmitter antennas as

$$
C_{k}=\frac{1}{2} \log \left(1+\sigma^{-2} P_{k} \max _{m=1, \ldots, N_{T_{k}}}\left\|\mathbf{h}_{k, m}\right\|^{2}\right)
$$

For antenna selection, the spatial signature of each user can be chosen only among the set of spatial signatures of the transmitter antennas, $\left\{\sqrt{P_{k}} \mathbf{h}_{k m}\right\}$. Following the same approach as in the eigenmode selection, intuitively, an effective antenna selection method is to choose the transmitter antenna that will maximize the received power of the user, i.e., the transmitter antenna with the highest norm $\left\|\mathbf{h}_{k m}\right\|$. In this case, the spatial signature of the user is

$$
\mathbf{a}_{k}=\sqrt{P_{k}} \underset{\left\{\mathbf{h}_{k m} \mid m \in\left\{1,2, \ldots, N_{T_{k}}\right\}\right\}}{\arg \max }\left\|\mathbf{h}_{k m}\right\|
$$

The scheduling algorithm with maximum received power antenna selection (MAXAS) is the algorithm presented in Table I with the effective spatial signatures defined as the spatial signatures of the transmitter antennas with the highest norm.

This approach is expected to perform well if one transmitter antenna has a spatial signature whose norm is significantly larger than the others, and the MIMO channels of the users are independent of each other. If this is not the case, a similar approach to the generalized eigenmode selection that compares the performances of all transmitter antennas in terms of minimizing the performance difference from a single user's perspective, can be devised. We term this algorithm the scheduling algorithm with generalized antenna selection (GENAS). The resulting scheduling and antenna selection algorithm is a two-step algorithm where the best transmit antennas are found at the first step, and the user with the highest $z_{i k}$ is assigned a time slot next.

\section{NumericAl Results AND CONCLUSiON}

In this section, we present numerical results related to the performance of the scheduling algorithms. We also compare the performance of the scheduling strategies for different levels of feedback to investigate the benefit gained by exploiting the CSI. The simulations are performed for a multiuser MIMO scheduler with $N=8$ time slots with $K=16$ users. The channels are realizations of a flat fading channel model where all links are assumed to be i.i.d. complex Gaussian random 


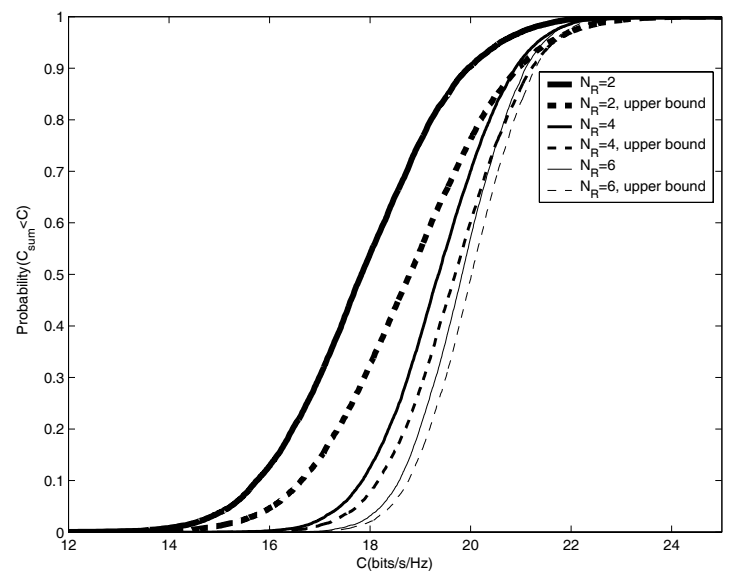

Fig. 1. $K=16$ user MIMO system with single transmitter and $N_{R}=$ $2,4,6$. Comparison of the multiuser MIMO scheduling algorithm.

variables. The received SNR of each user is $7 \mathrm{~dB}$. CDF curves for sum capacity obtained by simulating 10000 channel realizations are presented.

First, we consider a $K=16$ multiuser MIMO scheduler with single transmit antenna per user and $N_{R}=2,4$ and 6. The upper bounds, and the performance of the proposed scheduling schemes presented in Table I, are given in Fig 1. We observe that the proposed scheduling algorithm achieves sum capacities very close to the upper bounds. As we increase the number of the receiver antennas, the performance of the scheduling algorithm is enhanced. This is expected since for each added receiver antenna, the spatial diversity increases, and the users become less correlated.

We compare the performance of the scheduling strategies for $K=16$ user $4 \times 4$ MIMO system with different levels of feedback to investigate the benefit gained by exploiting the CSI in Fig 2. As expected, the performance of the algorithms is improved as the level of feedback is increased and the scheduling algorithm with perfect transmit beamformer feedback (PTBF) performs the best. Notice that the generalized beamformer design that considers the performance of all transmitter antennas/eigen modes with scheduling (GENAS/GENES) outperforms the maximum received power antennas/eigen modes selection approach (MAXAS/MAXES). However, we note that the largest relative gain is obtained when the feedback related to each user's own CSI is available, which enables each user to select its transmit beamforming vector so that its received power is maximized. We also observe that scheduling with antenna selection, which does not consider the best (single or multi user) eigen modes to transmit, performs relatively poorly.

The numerical results demonstrate that as the level of feedback increases, the performance of the proposed scheduling scheme improves and comes close to the upper bound. The individual CSI provides a substantial capacity gain due to the fact that the individual received powers can be maximized by channel information at the transmitter. As expected, the gap between the performance of the proposed algorithm, and the upper bounds decreases as the dimension of the MIMO system is increased.

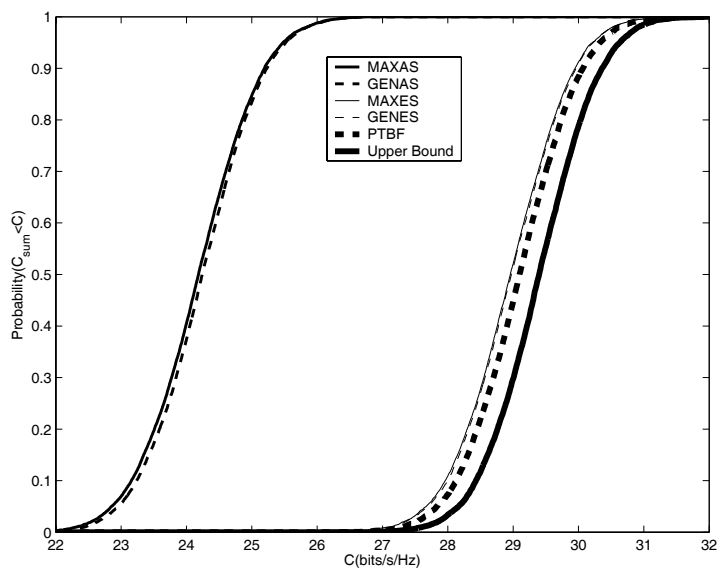

Fig. 2. $K=16$ user system with $4 \times 4$ MIMO model. Comparison of the multiuser MIMO scheduling algorithm with different levels of feedback.

In conclusion, in this paper, we considered the problem of designing scheduling algorithms for multiuser MIMO systems with low complexity that achieve near-optimum sum capacities. As the problem of finding the optimum scheduler is NPcomplete, we have taken the approach of designing schedulers with good heuristics that perform close to the sum capacity upper bound. Since the performance of the scheduler depends on the transmit beamformers, we have considered transmit beamformer selection next, and proposed several methods with different levels of feedback at the transmitter side. We have observed that as the feedback level at the transmitter is increased, the performance of the proposed algorithms approaches to the capacity upper bounds, and consequently to the capacity of the optimum scheduler. Notably, the individual CSI feedback facilitates a substantial gain.

\section{REFERENCES}

[1] E. Telatar. Capacity of multi-antenna Gaussian channels. European Tran. on Telecomm., 10(6):585 - 595, Nov./Dec. 1999.

[2] V. Tarokh, N. Seshadri, and R. Calderbank. Space-time codes for high data rate wireless communication: performance criterion and code construction. IEEE Tran. on Info. Theory, 44(2):744-765, March 1998.

[3] H. Sampath, P. Stoica, and A. Paulraj. Generalized linear precoder and decoder design for MIMO channels using the weighted MMSE criterion. IEEE Tran. on Comm., 49(12):2198-2206, Dec. 2001.

[4] S. Serbetli and A. Yener. Transceiver optimization for multiuser MIMO systems. IEEE Tran. on Signal Processing, 52(1):214 - 226, Jan. 2004.

[5] Lai-U Choi and R. D. Murch. A transmit preprocessing technique for multiuser MIMO systems using a decomposition approach. IEEE Tran. on Wireless Comm., 3(1):20 - 24, Jan. 2004.

[6] R. Zhang. Scheduling for maximum capacity in S/TDMA systems. In Proc. IEEE International Conf. on Acoustics, Speech, and Signal Processing, pages 2141-2144, 2002.

[7] S. Verdú. Capacity region of Gaussian CDMA channels: The symbolsynchronous case. In 24th Annual Allerton Conf. on Communication, Control and Computing, pages 1025-1034, Oct. 1986.

[8] P. Viswanath and V. Anantharam. Optimal sequences and sum capacity of synchronous CDMA systems. IEEE Tran. on Info. Theory, 45(6):1984-1991, Sept. 1999.

[9] S. Serbetli and A. Yener. Time-slotted multiuser MIMO systems: Beamformer and scheduling strategies. EURASIP JWCN, Special Issue on Multiuser MIMO Networks. Accepted for publication. Available at http://labs.ee.psu.edu/faculty/yener/publications.html.

[10] Rick S. Blum and Jack H. Winters. On optimum MIMO with antenna selection. IEEE Comm. Letters, 6(8):322-324, August 2002.

[11] A. F. Molisch, M. Z. Win, and J. H. Winters. Capacity of MIMO systems with antenna selection. In Proc. International Conf. on Comm., 2001. 